\title{
The Conservative Approach to Dental Crown Fractures in Dogs and Cats
}

\author{
Raluca-Ioana NEDELEA ${ }^{1 *}$, Adrian TOMA ${ }^{2}$, Andreea MARINCUS ${ }^{3}$ \\ ${ }^{1}$ Doctor of Dental Surgeon, DentOralMax, Cluj-Napoca, Romania \\ ${ }^{2}$ Doctor of Veterinary Medicine, Clinica Calvaria, Cluj-Napoca, Romania \\ ${ }^{3}$ Doctor of Veterinary Medicine, Clinica Calvaria, Cluj-Napoca, Romania \\ *corresponding author: Raluca.Nedelea@dentovet.ro
}

Bulletin UASVM Veterinary Medicine 76(2)/2019

Print ISSN 1843-5270; Electronic ISSN 1843-5378

doi:10.15835/buasvmcn-vm:2019.0023

\begin{abstract}
:
Dental crown fractures in small animal pathology are common dental issues in every day practice. The most frequently performed procedure is extraction with all the negative consequences.

The conservative approach to dental crown fractures involves endodontic treatment and coronal reconstruction in order to keep the fractured teeth on the dental arch.

They were taken into study 74 dental crown fractures involving the pulp chamber, leaving the root undamaged.

Clinical and radiological one-year check-up revealed us that the conservative approach to dental crown fractures in dogs and cats, when the selection criteria correspond to the indications of the specialized literature and when it is performed by latest protocols, is a reliable alternative to veterinary dental extractions.
\end{abstract}

Keywords: cat, dental fractures, dog, endodontic treatment, dental crown

\section{Introduction}

Oral pathology in cats and dogs occupies first place in their morbidity, but it is the most underdiagnosed and undertreated veterinary medical issue. Dental crown fractures occur as a consequence of a mechanical trauma when a determined force is applied on dental structures in a variable angle. Bite force of one individual is a major factor in this complicate equation. Bite force, according to Kim et al. (2018), is in a direct relationship with body weight, patient's skull, size and morphology. In order to establish which types of teeth are most commonly fractured, we have to corroborate the geometry of the dental arch with the every day activity of our patients. Cats, usually fracture their canines, due to their exposure in the dental arch in failed landings after jumping or cats' fights. Dogs as they are more dynamic and have a greater biting force will present a wider range of teeth' types that are fractured.

According to Reiter et al. (2018), the maxillary fourth premolar tooth of a dog has a higher incidence of fracture compared to any other teeth. The most wide spread procedure for treating dental fracture is tooth extraction. There are several potential complications during and after surgery that may happen. Even if well performed a lot of inconvenient local consequences can occur: extensive bone loss, adjacent teeth losing their supportive periodontium and bone, so that they will acquire more easily periodontal mobility and periodontal pockets, bending towards the new free space thus resulting premature, unphysiological contacts between teeth and gingiva and we have to mention the injury produced by the direct impact 
of food on the remaining gingiva on the edentate space. It is important to understand that every dental unit lost represents a severe functional impairment for the oral cavity, for the dental occlusion's harmony, for the temporo-mandibular joint and even more, for the whole process of mastication with a lot of negative consequences on digestion.

The conservative approach to dental crown fractures consists of endodontic treatment and coronal reconstruction in order to keep the fractured teeth on the dental arch, avoiding the above-mentioned complications.

\section{Materials and methods}

We took into study only dental crown fractures that involved the pulp chamber, with no root fractures, treated by over a period of oneyear time, from the $1^{\text {st }}$ of January 2018 to the $31^{\text {st }}$ of December 2018, from Romania, Transylvania area. All cats and dogs were in complete state of health confirmed by biochemical blood tests and a thorough clinical medical examination. Radiographs were taken to ensure lack of periapical processes and to establish working length.

Diagnostic of dental crown fracture was based on inspection, as a fractured tooth has sharp enamel edges, always in comparison with the symmetric tooth from the contralateral side. When the pulp chamber is affected, one can see a pink-reddish point, on the fractured surface, when the fracture is recent, or a dark-black point

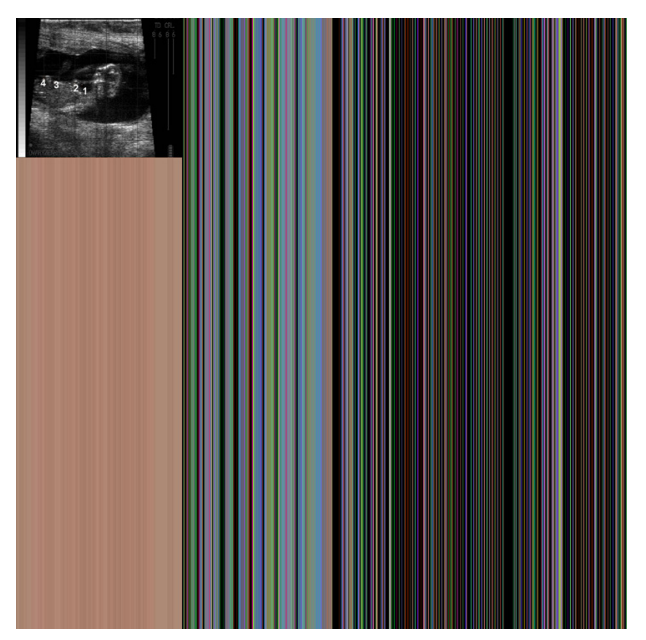

Figure 1. Dental probe exploring the opening of the pulp chamber of a fractured 303 in a dog, personal archive if the fracture has occurred earlier in time. When exploring with a dental probe the fractured area, the instrument will sink into the pulp chamber through that coloured point, mentioned before. (Reiter et al., 2018).

A number of 9 cats: 5 males and 4 females and 52 dogs: 23 bitches and 29 males, were selected by the criteria to be included into study. The age of the subjects varied from 9 months to 10 years in dogs, and from 2 years to 7 years in cats. Regarding the fractured teeth's type on species, we had to treat 9 felines with 6 upper canines and 3 lower canines.

In the 52 dogs studied there were fractured: 16 upper incisors, 7 lower incisors, 9 upper canines, 10 lower canines, 3 upper premolars, 2 lower premolars, 10 upper carnassials, 8 lower carnassials. (Maxillary fourth premolar and first lower molar are considered carnassials in dogs).

All dental procedures were performed under general anesthesia associated with local nerve blocks, according to the clinical situation. Endodontic treatment followed the latest endodontic protocols. Standard root canal treatment was performed aseptically. Working length was established radiologically and electrically. In the selected cases a mechanical and chemical debridement of the root canals was performed, with abundant endodontic irrigations, with hypochlorite $5.25 \%$ after each endodontic file and the last irrigation was made with physiological serum, (Moldoveanu, 2018). Root canal filling was made by cold lateral condensation technique in

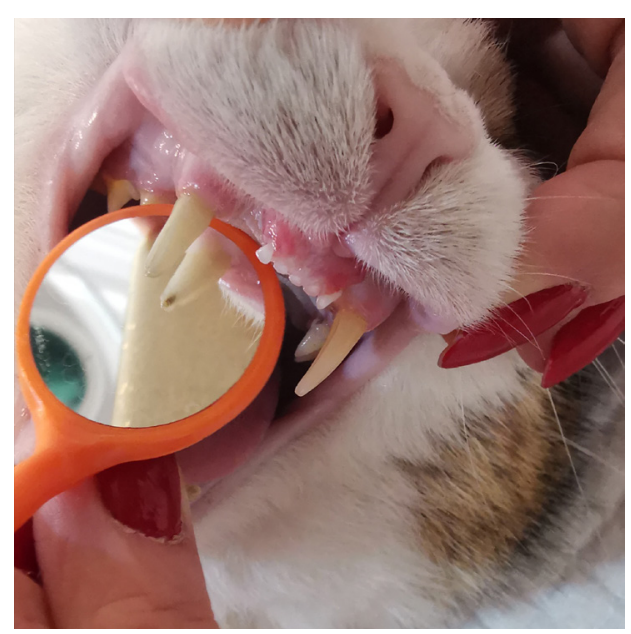

Figure 2. Image of complicated crown fracture of a 104 in a cat, personal archive 


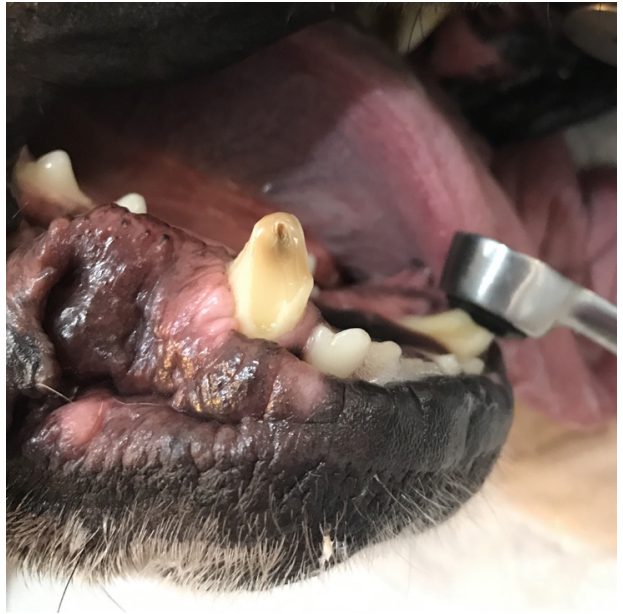

Figure 3. Image of complicated crown fracture of a 404 in a dog, personal archive

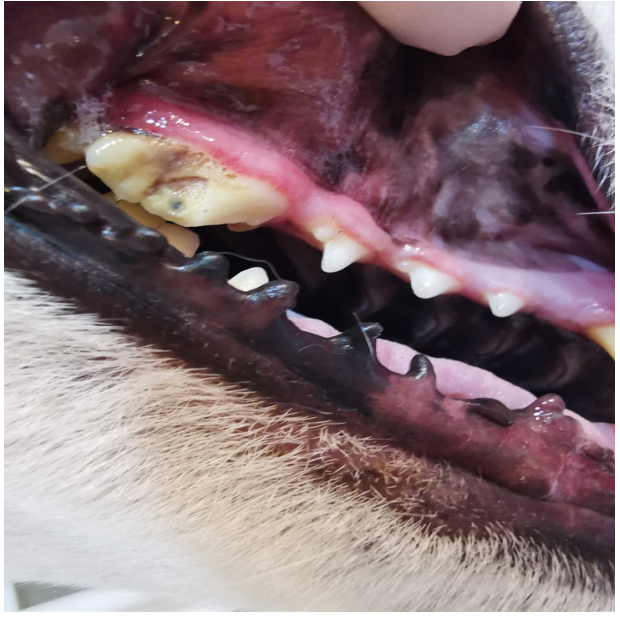

Figure 4. Tooth 108 presenting a complicated crown fracture in a dog, personal archive

\begin{tabular}{|c|c|c|c|c|c|c|c|c|c|c|c|c|c|c|c|c|c|c|c|c|c|c|c|c|c|}
\hline Age / Gender & 1/F & $1 \mathrm{M}$ & $2 \mathrm{~F}$ & $2 \mathbb{M}$ & $3 / F$ & $3 \mathrm{M}$ & 4/F & $4 \mathrm{M}$ & $5 / F$ & 5/M & 6/F & 6/M & $7 / \mathrm{F}$ & $7 \mathrm{M}$ & $8 / F$ & $8 \mathrm{M}$ & 9/F & 9/M & 10/F & $10 \mathrm{M}$ & Total F & Total M & Total & Total tipe & Tipe \\
\hline Upper Incisors & & & 4 & 1 & 1 & & & & & 2 & 2 & & 4 & 1 & & & & 1 & & & 11 & 5 & 16 & \multirow{2}{*}{23} & \multirow{2}{*}{ Incisors } \\
\hline Lower Incisors & & & & & 3 & 1 & & & & 2 & & & 1 & & & & & & & & 4 & 3 & 7 & & \\
\hline Upper Canines & & & 1 & 3 & & & & & & 1 & & & 1 & 1 & & & 1 & 1 & & & 3 & 6 & 9 & \multirow{2}{*}{19} & \multirow{2}{*}{ Canines } \\
\hline Lower Canines & 1 & & 1 & 1 & & & & & & 4 & & & & 1 & & & & 2 & & & 2 & 8 & 10 & & \\
\hline Upper Premolars & & & & & & & & & & & & & 1 & 1 & & & & & 1 & & 2 & 1 & 3 & \multirow{2}{*}{5} & \multirow{2}{*}{ Premolars } \\
\hline Lower Premolars & & & & & & & & & & & & & & 1 & & & & & & 1 & 0 & 2 & 2 & & \\
\hline Upper Carnassials & & & & 1 & & & & & 1 & & 1 & & 3 & 1 & & & & & 1 & 2 & 6 & 4 & 10 & \multirow{2}{*}{3} & \multirow{2}{*}{ Carnassials } \\
\hline Lower Carnassials & & & 1 & 1 & & & & & & 1 & & & 2 & & & & & 1 & & 2 & 3 & 5 & 8 & & \\
\hline Total & 1 & 0 & 7 & 7 & 4 & 1 & 0 & 0 & 1 & 10 & 3 & 0 & 12 & 6 & 0 & 0 & 1 & 5 & 2 & 5 & 31 & 34 & 65 & 65 & \\
\hline
\end{tabular}

Figure 5. Table with our data base of dogs' fractured teeth

order to obtain a complete, dense obturation of the entire root canal. Dental materials used for endodontic sealing of the root canals were guttapercha and mineral trioxide aggregate (MTA). MTA is a well-known endodontic material, a powder mixture of calcium silicates comprised of calcium oxide and silicon dioxide and a mixing liquid containing aqueous solution of calcium chloride and polycarboxylate. MTA has an excellent sealing ability, antibacterial effect (especially against Enterococcus faecalis and Streptococcus sanguis), well tolerated by the tissues, with a good biocompatibility and a recognized regenerative property. MTA does not react in contact with other restorative materials, thus yielding all the needed properties for an endodontic sealant with a good prognosis. Radiographs were taken to confirm proper root canal obturation.

Every endodontic treatment is considered with a good prognosis in time, when coronal restauration manages to seal it, preventing bacterial invasion to the tip of the root and the bone around it. Microleakage is one of the main reasons in endodontic failures. (Hargreaves et al., 2016).

When choosing the type of coronal reconstruction, one should consider the lost amount of dental structures. Small amounts of coronal volume may be replaced by coronal fillings made with light curing materials. The indication for prosthodontic reconstruction, involving a dental laboratory is imperative when a significant part of the coronal volume is lost. (Hargreaves et al., 2016). Coronal fillings' application technique followed producer's indications, using a lightcured composite restorative material (G-aenial Universal, GC Europe). Our used light-cured composite is made of diverse multifaceted structures and a variety of particles consisting of micro-filled matrix of resin composite, with 


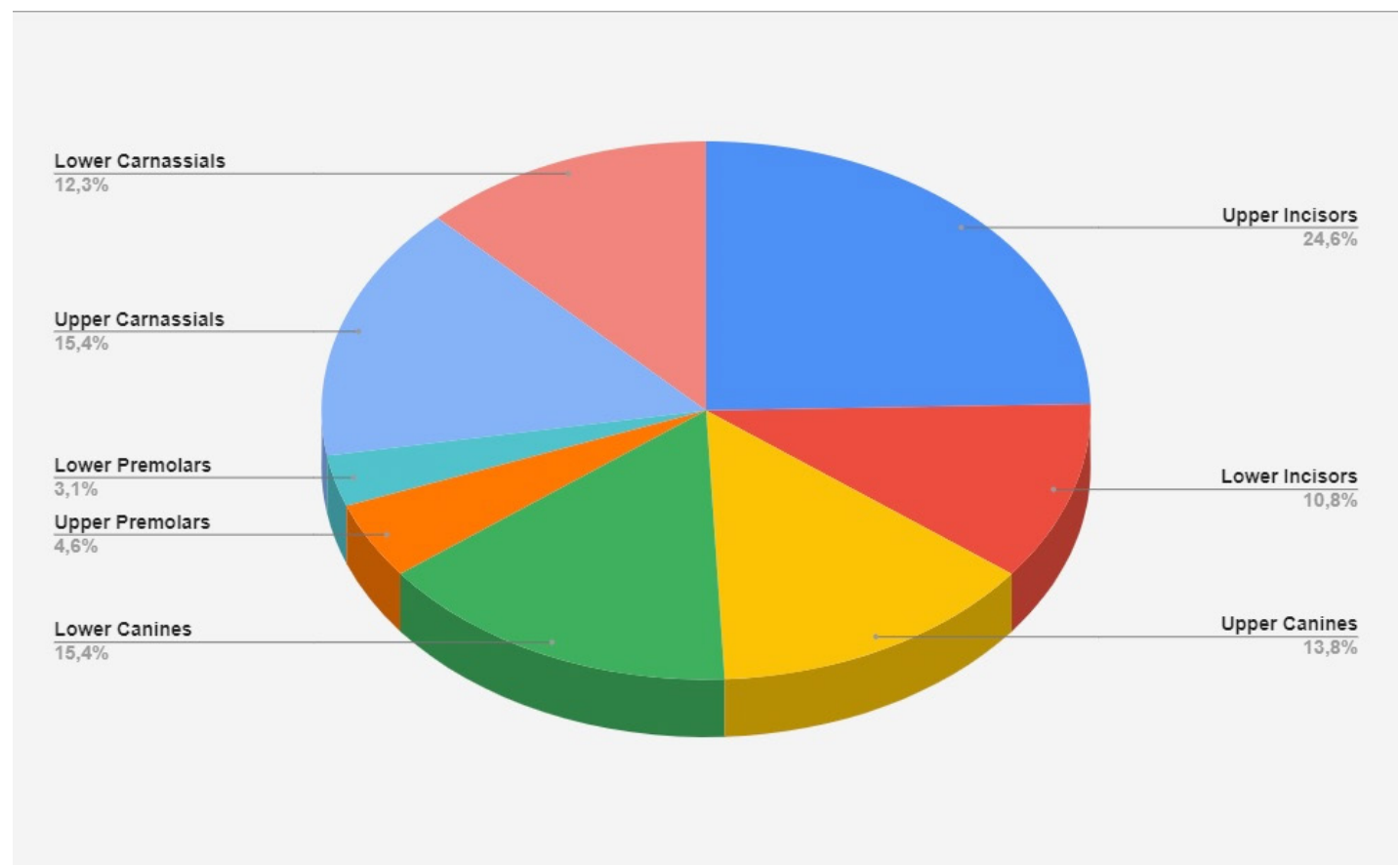

Figure 6. Distribution of fractured teeth in dogs

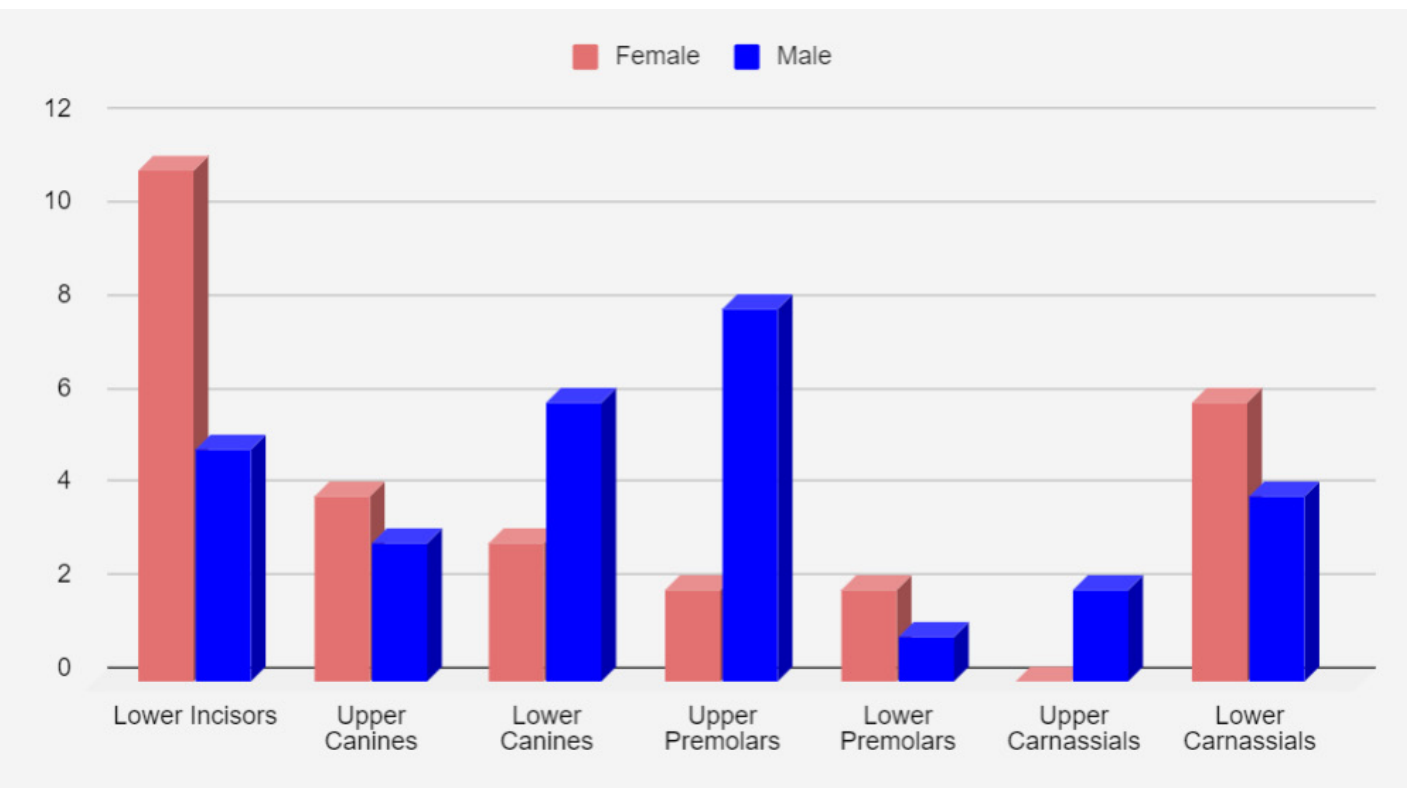

Figure 7. Repartition on gender and age of fractured teeth in dogs

compounds mainly of silica and pre-polymerized fillers. First of all, in order to degrease and to remove any residuals it is applied a conditioner gel, with $37 \%$ orthophosphoric acid, for 20 seconds. Then it is flushed it out with clear water and after the area is dried smoothly with the air syringe for about 20 seconds. The goal is accomplished when one could clearly see a white-snowy, chalky like enamel area, with no glaze around our coronal preparation. A 2 step $5^{\text {th }}$ generation bonding agent (Single Bond 2-3M, USA) was then applied and light cured for 20 seconds. Previous lackingin glaze surfaces will transform themselves in glossy ones. The composite is placed in sequential layers of $2 \mathrm{~mm} / 20$ seconds of light curing in order to achieve a good polymerization and a volume stability in time (Roman, 2000). 


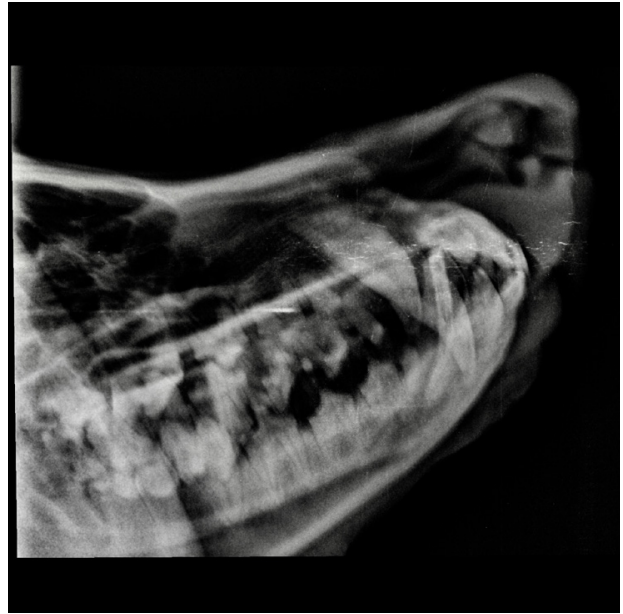

Figure 8. Radiography of a 404 endodontic treatment in a dog, personal archive

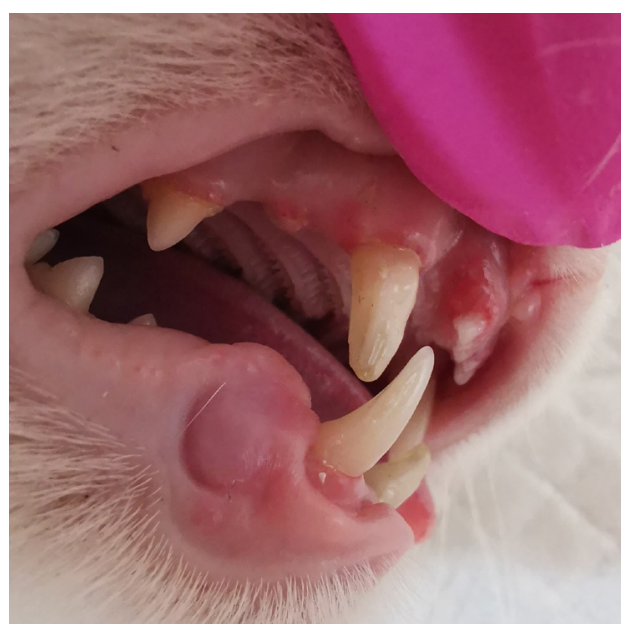

Figure 10. Image of a coronal recontruction with ligh curing materials in a cat, personal archive

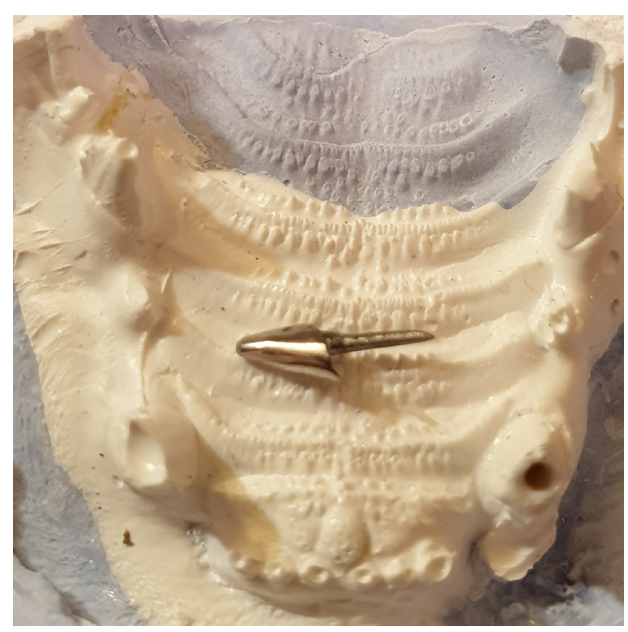

Figure 12. Image of a metal alloy Richmond type crown for an upper canine in a cat, personal archive

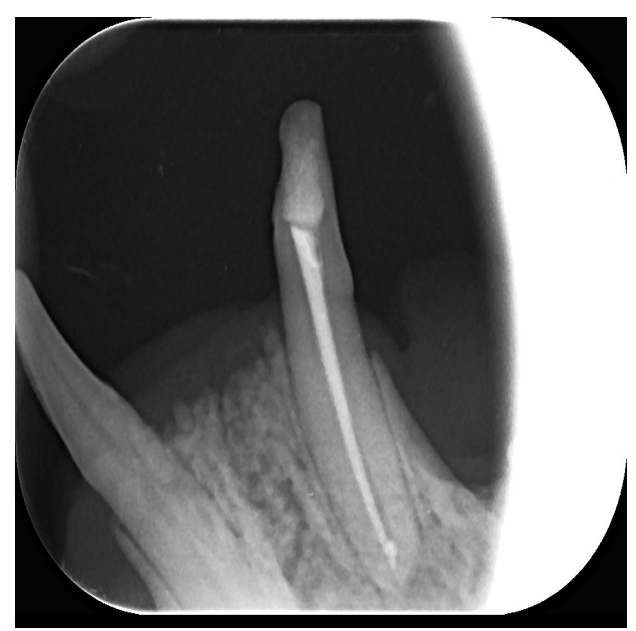

Figure 9. Radiography of a 404 endodontically treated, in a cat, personal archive

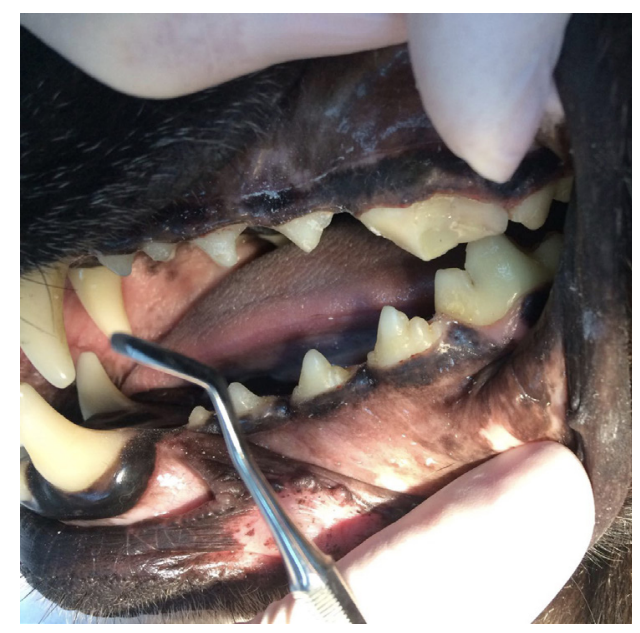

Figure 11. Image of a light curing filling made on an upper 4th maxillary premolar, personal archive

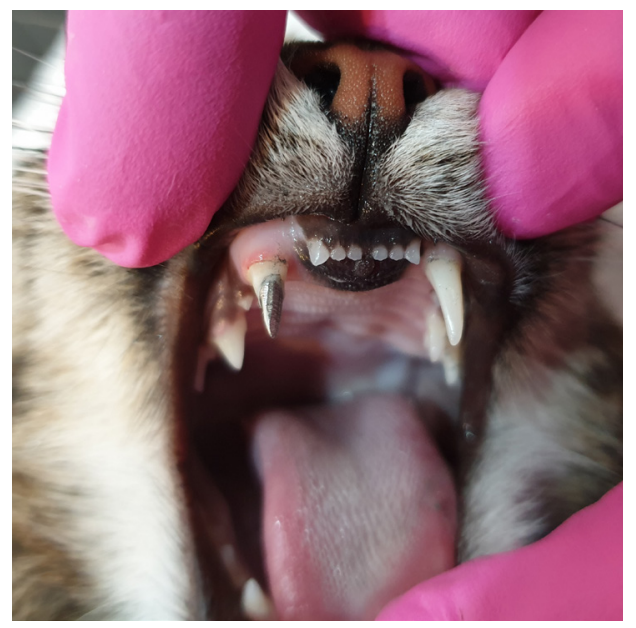

Figure 13. Image of the matal alloy reconstructed upper canine in a cat, personal archive 


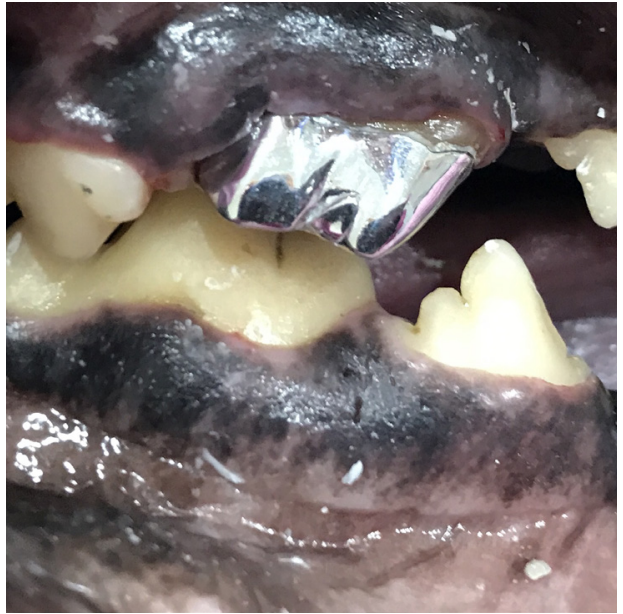

Figure 14. Image of a metal alloy reconstructed upper carnassial, personal archive

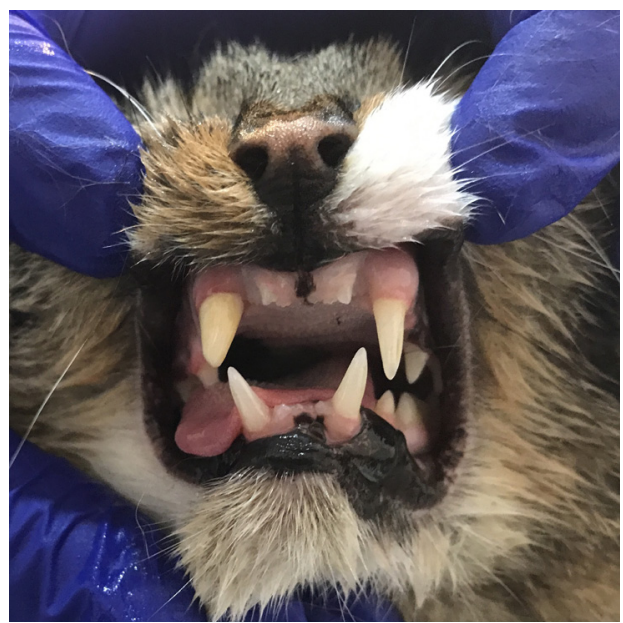

Figure 16. Image of a zirconia reconstruction of a canine in a cat, personal archive

All prosthodontic crowns were realized as Richmond type crowns, to ensure stability in time. Richmond type crown increases the length of the remaining crown, managing the arch of rotation under oblique forces. Richmond type crown is indicated in situations when there is an extensive loss of dental structures associated to deep bite, with no or less overjet, like in veterinary dentistry (Mishra, 2015). In order to obtain a Richmond type prosthodontic piece, one should prepare the root similar to the preparation for a dowelcore: $1 / 3$ of the root diameter and $2 / 3$ of the root's length, leaving at least $4 \mathrm{~mm}$ of endodontic treatment. An impression is taken in order to be sent to the dental laboratory. We made a two-step

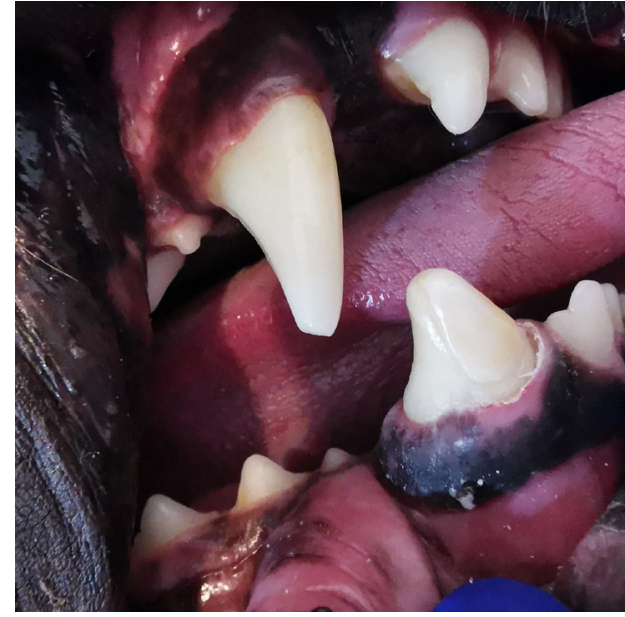

Figure 15. Image of a zirconia reconstruction of a lower canine in a dog, personal archive

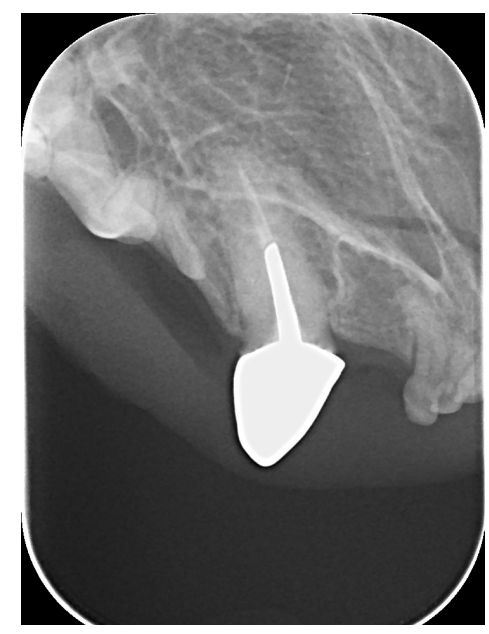

Figure 17. Radiography made at one year follow up for an 104 reconstructed with a Richmond type crown, in a cat, personal archive

impression technique with condensation silicones with a stability up to 7 days. (Speedex, Coltene Europe). Two-step impression techniques involves the use of a heavy body and when the material is set, the second step is to wash the impression with a light body impression material in order to obtain the necessarily details. Our light body materials have hydrophilic properties, thus being able to ensure perfect detail reproduction even in a moist environment. Dental laboratory made our prosthodontic pieces with computer-aideddesign/computer-aided-manufacturing (CAD/ CAM).

Before cementing we have tested the marginal closing, bite's fitting, the right manufacturing. 
After that we degreased both the prosthodontic crown and the tooth structures. The luting cement that we have used was a resin modified glass ionomer that provides the highest level of confidence through long term clinicals (Fuji Plus, GC Europe). It has chemical adhesion to the tooth structures ensuring an excellent marginal seal, a moisture tolerant solution when a dry field cannot be assured with no bonding procedures needed. Rubbery consistency makes easy the removal of the excess. It is a multipurpose luting cement, being able to cement metal-based, zirconia, alumina and resin restorations.

Zirconia stabilised with yttrium is an innovative prosthodontic material, with mechanical properties as stated by the producers, similar to metal alloy, but more aesthetic and more biocompatible than it (Cousland et al., 2018). The only inconvenient issue with zirconia would be the price, as it has higher costs of production.

Based on the coronal reconstruction, after endodontic treatment, repartition on species we report in felines: 6 coronal fillings with light curing materials, and 3 prosthodontic crowns: 2 made of metal alloy and one of zirconia stabilised with yttrium. Dogs received 61 coronal fillings, made with light curing materials, and 2 metal alloy prosthodontic crowns and 2 made of zirconia stabilised with yttrium.

\section{Results and discussions}

Our results revealed that, in our selected population, most fractured teeth are incisors, followed by canines and on the $3^{\text {rd }}$ place carnassials. However, our study has its limitations as, few people know and are aware of the dental care importance, in our country and county. First step is to educate the owners and medical stuff to recognize and to prevent oral issues in small animals. Dental care for veterinary patients has to become a priority, as oral pathology may lead to complications in vital organs and decreases the quality of life for our patients.

One-year follow-up, clinical and radiological, revealed no periapical lesions, no crown loosening and only 3 coronal fillings missing, in dogs.

Good diagnosis leads to good treatment procedure with a good prognosis. Once established that we are dealing with a complicated crown fracture, one should perform an aseptically standard root canal therapy, throughout the whole working length. Endodontic treatment is a minor surgical procedure with a lot of failure-susceptible stages, and one should be up to date with the latest endodontic protocols knowing exactly when and where to pay increased attention in order to obtain repeatable and sustainable results.

Coronal sealing of the endodontic treatment is a major step in order to obtain long term results. Knowing exactly when to perform a prosthodontic coronal reconstruction is also a key to success, but it requires high level abilities from the dental laboratory, as we all know a veterinary dentistry course for dental technicians does not exist.

Educating owners in posttreatment playing patterns will ensure us a higher percent of success. Our 3 coronal missing fillings are based on continuing playing with wooden cracks or ropes and owners not being able or not paying enough time to unlearn bad habits.

\section{Conclusions}

No matter the age, the gender and species when selected criteria correspond to the indications of the specialized literature, the conservative approach to dental crown fractures in cats and dogs is a reliable alternative to veterinary dental extractions. Modern dental techniques associated to latest dental materials ensure a good prognosis. Creativity and knowledge of the doctor has to meet the possibilities of the dental laboratory and the willingness of the owners to invest time and money in their pets.

An interdisciplinary approach, where human science meets the veterinary medicine will lead us to great accomplishments on both sides.

Acknowledgements. This work did not receive any specific grant from funding agencies in the public, commercial, or not-for-profit sectors.

\section{References}

1. Reiter AM, Gracis M (2018). Canine and feline dentistry and oral surgery. ( $4^{\text {th }}$ ed.). Gloucester: BSAVA, (Chapter 9).

2. Kim SE, Arzi B, Garcia TC, Verstraete FJM (2018). Bite forces and their measurement in dogs and cats. Frontiers in veterinary dentistry. DOI: 10.3389/fvets.2018.00076

3. Mishra P, Mantri SS, Deogade S, Gupta P (2015). Richmond crown: a lost state of art. International journal of dental and health sciences. 02. 448-453.

4. Moldoveanu B (2018). Materiale bioceramice in endodontie. Aceeasi diferenta? Dentalife.102.6-9. 
5. Hargreaves KM, Berman LH (2016). Cohen's pathways of the pulp. (11 $1^{\text {th }}$ ed.). Missouri: Elsevier, (Chapter 22).

6. Roman A, Pop A (2000). Caria dentara de la teorie la practica. ( $1^{\text {st }}$ ed.). Cluj-Napoca: Editura Dacia, (Chapter 13).
7. Cousland G, Cui XY, Smith A, Stampfl APJ, Stampfl C (2018). Mechanical properties of zirconia, doped and undoped yttria-stabilized cubic zirconia from first-principles. Journal of Physics and Chemistry of Solids. 122.10.1016/j. jpcs.2018.06.003. 